\title{
Il Teatro in Dante: lo spettacolo dell'invidia
}

\author{
Deirdre O'Grady \\ University College Dublin \\ deirdre.ogrady@ucd.ie
}

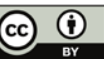

\section{Riassunto}

Il teatro come forma d'arte consiste di un'azione creata da un autore e animata da attori in presenza di un pubblico osservatore. Il Canto XIII del Purgatorio si svolge attraverso strumenti linguistici che si trasformano in effetti drammatici identificabili con una messa in scena moderna. La descrizione scenica, le luci riflettori e gli effetti uditori servono a toccare l'essenza del canto dei penitenti invidiosi. Si svolge uno spettacolo dell'Invidia del "vedere" e "non vedere", dell'"osservare", "ascoltare", "sentire" e "partecipare". Questi verbi vengono utilizzati per realizzare il dramma centrale dell'episodio: un contrasto fra il "visto" e il "non visto", e l'immagine e il ricordo/confessione del personaggio principale Sapia di Siena. Il ricordo di lei crea una nuova realtà drammatica. L'azione viene controllata dall'autore che sdoppia la propria personalità in modo da commentare e conversare dall'interno del testo/palcoscenico. La "recita" coinvolge il pubblico/lettore e mette in evidenza la differenza e l'affinità fra la partecipazione di un pubblico in teatro e il leggere un testo drammatico.

Parole chiave: Divina Commedia; invidia; cecità; vista; osservazione; partecipazione; recitazione; interpretazione.

\begin{abstract}
Theatre as an art form consists of a series of actions created by a writer and animated by actors in the presence of an observing public. Canto XIII of Purgatorio develops with the aid of linguistic devices that are transformed into dramatic effects, identifiable with a modern performance. Scene description, lighting, and sound effects serve to highlight the essential aspects of the Canto of the repenting Envious. There evolves a spectacle of Envy - of 'seeing' and 'not seeing', of 'observing', 'listening' 'hearing' and 'participating'. These verbs are utilized in order to create the drama central to the episode: the contrast between the 'seen' and 'unseen' and the visual significance and memory/confession of the principal persona Sapia of Siena. Her recollection creates a new dramatic reality. The action is controlled/directed by the author who reinvents his own person in order to converse and comment from inside the text/stage. The 'performance' involves the reader/ public and highlights both the difference and affinity between a theatrical presence and a reading of a dramatic text.
\end{abstract}

Keywords: Divine Comedy; envy; blindness; vista; observation; participation; acting; interpretation. 


\section{Leggere il teatro: Osservazione e Partecipazione}

66 ante e il teatro", "Il teatro in Dante": due titoli che confermano il drammatico nell'opera dantesca e particolarmente nella Divina Commedia. Il primo identifica una presenza dantesca nel mondo del palcoscenico, il secondo riconosce nell'opera del nostro poeta una cura scrupolosa per le figurazioni sceniche e simboliche, per la descrizione, nonché per l'interazione drammatica tra i personaggi dell'oltretomba e i due poeti viaggiatori. Dunque gli episodi sono più che sequenze di narrazione letteraria, spesso costituiscono delle micro rappresentazioni di drammi ora religiosi, ora politici ora morali, che il lettore contemporaneo può facilmente collegare con il teatro più avanzato e moderno dei nostri giorni.

Dante ci presenta delle figure storiche viste attraverso i momenti più drammatici della loro esistenza, circondate da immagini e scene simboliche che facilmente si identificano con una scenografia moderna. Lo scopo di quest'articolo è dimostrare come il Canto XIII del Purgatorio, il canto di Sapia, sia dominato dalla presenza della "teatralità" che si esplica attraverso il valore scenico e simbolico dell'illuminazione e della vista, la recitazione e la gestualità dei personaggi, con il risultato finale di portare lo spettatore da un piano fisico attivo a uno psicologico contemplativo.

Non possiamo tralasciare l'importanza e l'influenza che l'opera dantesca ha esercitato su un gran numero di capolavori della drammaturgia di tutti i tempi. Il periodo romantico è in particolar modo ricco di opere teatrali le cui protagoniste femminili, create dal genio di Dante, acquistano nuovi valori e godono di una nuova fama. Mi riferisco in particolare a Francesca da Rimini, Pia de' Tolomei e Cunizza da Romano, creature che travalicano i loro limiti temporali e spaziali per assumere dei tratti storici e drammatici universalmente conosciuti ${ }^{1}$. È mia intenzione servirmi del teatro e dei suoi tecnicismi come metafora per un viaggio nell'arte dantesca. Per fare questo punterò su una lettura del testo focalizzata sulla tecnica linguistica e descrittiva del poeta e sul suo messaggio drammatico. Fondamentale è l'effetto sul lettore. Nell'esaminare aspetti specificamente teatrali noi lettori dobbiamo assumere un nuovo atteggiamento critico ed interpretativo. Per poter determinare quanto di teatrale è presente in Dante non ci accontenteremo della spiegazione filosofica e simbolica del "contrappasso"; rivolgiamoci invece agli "effetti" scenici, visivi e perfino psicologici che ci sono comunicati attraverso la parola scenica. Questo modo di esaminare le anime e la loro disposizione conferisce al lettore il ruolo di un pubblico osservatore, fisicamente presente, che interagisce

I. La storia dell'emancipazione della figura di Francesca da Rimini come figura letteraria romantica si trova in: O'Grady (2003), O'Grady (2013). In questa breve relazione si nota l'influenza della figura di Francesca sul teatro lirico. 
nell'episodio insieme a Dante pellegrino/viaggiatore. Attraverso il sopraddetto metodo critico constatiamo la presenza di un palcoscenico della vita. Dunque una visione "moderna" che getta nuova luce sulla tecnica drammatica dantesca. Perché un tale atteggiamento critico? Perché scelgo proprio questo canto per identificare il teatrale in Dante? Sono domande centrali al discorso, ma sono facilmente spiegabili se si tiene conto che Dante, oltre a un gran numero di effetti scenici introduce anche l'elemento psicologico per dare una nuova e più profonda dimensione all'azione.

Per partecipare ad uno spettacolo teatrale è necessaria la presenza fisica di un pubblico che segue l'azione da "vicino" e dal "vivo". Il pubblico quindi diventa osservatore del dramma. E questo conduce ad un rapporto con gli attori che creano l'azione ${ }^{2}$. La comprensione del dramma dipende dalla capacità del pubblico di vedere e sentire. Gli elementi visivi e uditivi devono funzionare in modo che le azioni di scena siano osservate e sentite. A questo scopo sono necessarie le luci di riflettori per illuminare la scena.

Mi pare che un'osservazione critica del canto degli invidiosi dal punto di vista del lettore/osservatore sia particolarmente rilevante per il nostro discorso, perché mette in luce l'impiego di certi effetti scenici e drammatici. Chi segue un'opera teatrale, vi partecipa con la vista e l'udito. Il lettore di Pg. XIII nella sua visione creativa del testo "vede" con l'occhio della mente la scena descritta e "sente" i diversi registri sonori che vengono usati: parlo delle voci volanti, la recitazione contenuta in un monologo drammatico e la presenza di un coro. La descrizione poetica dell'autore e l'osservazione critica del lettore si contrappongono allo stato di cecità delle anime "in scena". Infatti la forza drammatica del canto intero risulta dal contrasto fra vista e cecità nonché dal già indicato dramma psicologico del personaggio visto che non vede. L'importanza di questo momento poggia sulla sottile presenza della psicologia. Il canto è privo dei colori simbolici adoperati da Dante nella Valle dei Principi, né vi è traccia dell'intimità poetica dell'incontro con Forese o del simbolismo drammatico e teologico della processione mistica nel Paradiso terrestre. Al contrario, si coglie l'impressione di un dramma avvolto nell'oscurità. Dunque un senso dell'astratto che introduce una dimensione intellettuale che si avvicina a una stesura moderna. Per tutti questi motivi il canto di Sapia si realizza come un canto di una teatralità sottile e raffinata per eccellenza.

In scena sono gli invidiosi, paragonati a dei mendicanti ciechi che chiedono l'elemosina seduti sulle scale di una chiesa. L'episodio si sviluppa come un vero spettacolo drammatico, carico di ambiguità. È un canto in cui i temi della vista e dell'osservazione sono di prima importanza. Questi temi si tra-

2. Quest'aspetto del teatro è trattato dagli autori seguenti nei loro studi sul teatro e il posto della comunicazione sociale: Rossi-Landi (1979); Bentley (1965); Mounin (1985: 28-62). 
sformano in personaggi che esprimono la legge del monte. Viene così creato un dramma pieno di antitesi e contrasti basati sul significato della parola Invidia - il "non vedere" che si amplia nel corso del canto3.

La figura centrale del canto è Sapia di Siena ${ }^{4}$, l'unica donna identificabile del Purgatorio. Fisicamente è l'antitesi di Beatrice. Sapia simboleggia la mancanza delle qualità spirituali e intellettuali della "donna angelicata" della Vita nuova. La sua figura mette in rilievo il contrasto fra luce e ombra, guerra e pace, amore e odio, bene e male. È come se Dante proiettasse alla rovescia tutta la filosofia "stilnovista" in un contesto drammatico simbolico. La mancanza di movimento indica una mancanza di progresso morale dovuto all'aspetto paralizzante del peccato terreno. Dunque la tradizione scolastica medievale va capovolta nell'unica figura femminile identificabile del Purgatorio.

Nel canto centrale della Divina Commedia Pg. XVII si trova il ragionamento di Dante sulla teoria dell'amore come "semente... d'ogne virtute/e d'ogne operazion che merta pene". Gli invidiosi sono in possesso di un eccesso di amore per se stessi, al punto da non discernere la verità. In altre parole sono ciechi alla luce divina e di conseguenza "lividi per l'invidia" al punto da rallegrarsi della sfortuna degli altri. Già in If. XIII l'invidia è indicata come donnas:

La meretrice che mai da l'ospizio

di Cesare non torse gli occhi putti

morte comune e de li corti vizio,

infiammò contro di me li animi tutti

(If. XIII, 64-67)

Sapia, come personificazione dell'invidia, risulta una presenza poetica e drammatica priva di ogni segno di bellezza, giovinezza, carità e sapienza, qualità che si trovano personificate nella figura della "donna gentile" medievale $^{6}$. Nonostante il racconto carico di ironia e l'aspetto miserevole e insieme spaventoso delle anime, si assiste a un "dualismo dantesco": le prime parole pronunciate da penitente sono espresse in termini francescani:

"O frate mio, ciascuna è cittadina

d'una vera città;

(Pg. XIII, 94-95)

3. Si vedano i seguenti titoli nei quali si studia il canto degli invidiosi in maniera dettagliata: Della Terza (2013); Santini (I964); Singleton (1970); O’Grady, (1987) (la figura di Sapia è discussa nelle pagine 84-94).

4. Poca documentazione esiste sulla figura storica di Sapia. Si vedano: Sanesi (1926); Tempesti (1936).

5. La sorte della vittima dell'invidia viene considerata in confronto all'invidioso da Diana Glenn nel suo studio sulle donne in Dante: Glenn (2005).

6. O’Grady (1987: 73); Shapiro (1975); Donadoni (1963); Glenn (2008). 
L'immobilità delle anime penitenti indica una lentezza morale che si oppone alla violenza della vita politica attiva vissuta e ricordata più avanti nel canto. Il concetto di cittadinanza terrena sbiadisce in presenza dell'eterno. Così la scena drammatica si allarga, creando l'effetto di tempo spaziale in una terra abbandonata. Più avanti anime, figure, gesti e suoni sono rivelati sotto il Sole, enorme luce riflettore. L'apostrofe di Virgilio diventa sempre più significante quando si considera che il poeta latino simboleggia la ragione. Questa si lascia dirigere dalla rivelazione simboleggiata dal Sole. La luce fisica e simbolica è in contrasto con la cecità fisica e morale delle anime/personaggi/attori nello spettacolo dell'invidia. Una messa in scena precisa, studiata ma al tempo stesso capace di provocare reazioni spontanee ${ }^{7}$.

Dunque, come vedremo passando a un'analisi del canto, Dante, servendosi della parola poetica, realizza tutti gli effetti scenici e drammatici presenti in una regia teatrale moderna. $\mathrm{Ci}$ si rende conto dell'abilità del poeta toscano di creare "linguisticamente" ogni dimensione sia artistica, sia visuale sia sonora. Viene in luce il senso del "naturale" in Dante che si presta agli effetti scenici e ai gesti "artificiali" del mondo teatrale. L'applicazione di un metodo critico che mette in evidenza la vicinanza dell'opera dantesca a una drammaturgia più moderna non toglie niente all'originale; anzi mette in evidenza diversi aspetti artistici che potrebbero essere facilmente ignorati o persi. Così Dante proietta a prima vista un mondo francescano di povertà, penitenza e fraternità nel canto tredicesimo del Purgatorio. Ma Sapia di Siena in guisa di mendicante rivive drammaticamente la sua esperienza terrena. Latmosfera della lauda drammatica francescana di Jacopone da Todi si trasforma presto in un'esperienza realistica che ricorda il poeta Cecco Angiolieri. Ambedue gli atteggiamenti si esprimono attraverso una forza visiva evocativa. Siamo nel territorio di un dramma che coinvolge la vista, l'udito, e la memoria di un passato che si esprime e rivive in un presente poetico e drammatico. Dunque in un esercizio critico si contrappongono l'astratto e il fisico, il passato e il presente.

\section{DesCrizione SCENiCA E ILLUMINAZIONE SIMBOLICA}

Il canto si apre con una descrizione molto essenziale: il procedere in salita dei due pellegrini segue con precisione geometrica la forma circolare del monte. Si presenta all'occhio del lettore il mondo oscuro, livido e petroso dell'invidia mentre la solitudine del luogo richiama l'isolamento in cui è immersa la vita dell'invidioso:

Noi eravamo al sommo de la scala, dove secondamente si risega

7. La dottrina agostiniana della luce è trattata in: Jolivet (I933); Gardner (I9I3); Gilson (2000). 
lo monte che salendo altrui dismala.

(Pg. XIII, I-3)

parsi la ripa e parsi la via schietta

col livido color de la petraia.

(Pg. XIII, 8-9)

Luniformità della cornice e lo spazio sotto e sopra di essa creano l'impressione di stare su un palcoscenico moderno: grande, vuoto e completamente al buio. L'ingresso in scena del Sole mette in luce un nuovo elemento che emerge dall'ombra: un sentiero solitario sul quale Dante e Virgilio si avviano nei loro ruoli di Duce e Pellegrino, personaggi drammatici in un dramma della solitudine. Il "noi eravamo" descrittivo cede alla rettorica dell'Apostrofe con la quale Virgilio si rivolge al Sole:

"O dolce lume a cui fidanza i'entro per lo novo cammin, tu ne conduci" (Pg. XIII, I6-I7)

Il suono delle voci di spiriti invisibili che evocano atti di bontà, generosità $e$ amore introduce la parola recitata in sordina. A questo eco di voci fa seguito l'apparizione di anime penitenti con addosso mantelli dello stesso colore della pietra, che cantano la litania dei santi. Dunque una serie di suoni e immagini, fusi insieme, viene ad arricchire l'esperienza dei due poeti e del lettore. Sta per cominciare l'azione. A poco a poco lo spettatore si rende conto di presenze percepite con l'"udito" ma non con la "vista", e di altre presenze nella forma di ombre cieche percepite con la vista ma a loro volta incapaci di vedere. Il senso dell'udito pare amplificato. La voce del poeta latino proclama l'importanza del viaggio come missione. Il "dualismo dantesco" crea un contrasto fra mobilità e immobilità. Le anime non si muovono mentre Dante pellegrino avanza verso di loro in modo da farsi sentire.

Il ritratto degli invidiosi nel Canto XIII si sviluppa in tre momenti: il rendersi conto da parte di Dante della loro presenza, la loro descrizione fisica, l'imbarazzo di Dante che li "vede" non essendo però "visto" da loro. L'ultima di queste tre fasi apporta una dimensione psicologica al tema della vista e dell'osservazione. La reazione psicologica di Dante rappresenta un momento di distacco drammatico: un momento di accorgimento dello stato fisico delle anime. Questa reazione permette al lettore/spettatore di visualizzare lo spazio che separa il poeta dalle anime. Dante per ottenere un consiglio interpella Virgilio, che è diametralmente opposto al personaggio che sta per rivelarsi:

A me pareva, andando, far oltraggio, veggendo altrui, non essendo veduto:

(Pg. XIII, 73-74) 
Verso la fine del Canto l'anima di Sapia dichiara di essere consapevole del fatto che Dante è vivo, che egli non viaggia con gli occhi cuciti, e che pertanto è capace di "vederla" (vv. I30-32). Ormai il "vedere" e il "non vedere" assumono la forza di azioni astratte contenute in un testo drammatico.

Le parole di Dante indirizzate agli invidiosi esprimono la sicurezza di questi di "vedere" Dio - il Sole, descritto come "l'alto lume." Il dramma più attivo e centrale si sviluppa intorno a Sapia di Siena. Il suo gesto, il suo saluto, le sue parole e il suo commento conclusivo concorrono a creare due effetti diversi: il senso di penitenza che accompagna l'immagine delle anime e che inserisce quest' immagine in un quadro contemplativo; il momento contemplativo è seguito dalla recita drammatica di Sapia che la rende protagonista attiva di un episodio della vita terrena. Viene conferita alla figura una complessità drammatica basata su una poetica che comunica un doppio senso del visivo. Ricordiamo come la vista, il saluto, la luce e gli occhi formano la base della filosofia dell'Amore nella poesia del Dolce stil nuovo. Nella Vita nuova XXI, Sonetto II sono presenti le parole che mettono in rilievo la persona della donna gentile, alla quale Dante ha fisicamente contrapposto Sapia:

Ne li occhi porta la mia donna amore

Perché si fa gentil ciò ch'ella mira.

(Vn. XXI)

Nel Canto XIII del Purgatorio la Sapienza personificata da Virgilio è messa in contrasto con la Follia simboleggiata da Sapia. La Luce è messa in contrapposizione all'Oscurità. Questi simboli filosofici giocano le loro parti in un dramma del "visibile" e dell' "invisibile". L'effetto è di una forza drammatica tale da offrire al pubblico/lettore delle immagini da conservare nel teatro della mente.

\section{Il GeSTO DRAMMATiCo E la PAROla “GridATA”}

La terra livida e rocciosa è la seconda destinazione dei poeti. Le anime silenziose assomigliano a un coro operistico che si prepara ad intonare le prime note all'alzarsi del sipario. Ma non emettono nessun suono. Un coro muto dunque che ha il fine di introdurre una voce solista che grida un esempio della carità. L'anima della protagonista Sapia parla a lungo. Il suo ritratto prende forma attraverso un'impressione fisica che unisce i diversi aspetti del personaggio: la cecità, l'immobilità e la superbia invidiosa. È descritta con il mento in su, nell'atteggiamento tipico degli orbi:

Tra l'altre vidi un'ombra ch'aspettava in vista; e se volesse alcun dir "Come?" 
lo mento in guisa d'orbo in su levava.

(Pg. XIII, IOO-IO2)

Il gesto fra recitazione e mimo in quest'istante è indicativo di curiosità e di desiderio di parlare, che presto si trasformerà in un desiderio di sentirsi parlare, nonché di essere riconosciuta e rimessa in buona fama fra la sua gente. Il monologo drammatico è una recita contenente il suo ricordo degli eventi politici e storici del I269:

e veggendo la caccia,
letizia presi a tutte altre dispari,
tanto ch'io volsi in su l'ardita faccia,
gridando a Dio: "Ormai più non ti temo!"
come fé il merlo con poco bonaccia.
$(P g$. XIII, II9-I23)

Il gesto del viso volto in su si trasforma in un'espressione di gioia e di sfida che la accosta a Capaneo, colpevole di un atto di violenza contro Dio. La follia di Sapia viene associata con la follia nella favola del merlo ${ }^{8}$. Così la storia, la leggenda e la favola si uniscono per formare un dramma ad un tempo complesso e divertente. Interessante anche l'avventura del verbo "gridare", che sottilmente accompagna e interpreta il messaggio di Dante. Funziona come espressione drammatica, ed è di conseguenza parola che risponde a una necessità: il poeta è costretto ad alzare la voce giacché le anime si trovano ad una certa distanza da lui. La parola ad alta voce dimostra ardore. All'inizio del canto il verbo "gridare" ha la funzione del verbo "dire":

E prima che del tutto non si udisse per allungarsi, un'altra "I' sono Oreste passò gridando, e anco non s'affisse.

(Pg. XIII, 3I-33)

Le voci che si diffondono in scena recitano vari esempi di carità dal Nuovo Testamento: le parole di Maria alle nozze di Cana (Giovanni II, I-II), dal teatro greco antico, e dalle parole di Cristo (Matteo V, 24 e Luca VI, 27). Ciascun esempio risveglia nella nostra coscienza camei di gentilezza e di tenerezza. Il verbo "gridare" si trova là dove normalmente si usa "cantare" o "recitare" - infatti le animane "gridano" la Litania dei Santi in un impeto espressivo di ardore penitenziale. L'invocazione di Maria e dei Santi enunciata ad alta voce toglie il senso di pace che solitamente accompagna quelle parole. $\mathrm{Ma}$ l'intonazione forte fa anche ricordare che l'invidia è priva di sentimento e di sensibilità. Il grido conferisce un accompagnamento attivo alla loro presenza immobile:

8. Novella CXLIX del Trecento novelle (cfr. Pernicone (a cura di), 1946). 
E poi che fummo un poco più avanti, udia gridar: "Maria, òra per noi": gridar "Michele" e "Pietro" e "Tutti santi" (Pg. XIII, 49-5I)

Alla fine della narrazione di Sapia il "grido" si trasforma in insulto. Viene poi introdotto un altro registro drammatico quando ella lancia a Dio parole di disdegnoso orgoglio:

"Ormai più non ti temo!"

(Pg. XIII, I22)

Il gesto folle oltre a qualificare la stoltezza degli invidiosi, mette in luce anche la loro mancanza di giudizio. Solo le sante orazioni di Pier Pettinaio, terziano francescano, venditore di pettini, morto all'età di centonove anni, hanno reso

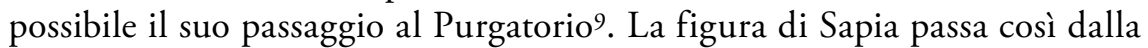
storia alla leggenda e dalla favola alla salvazione.

\section{LA Recita di SAPiA, CitTAdina SENESE GHIBELlina}

L'intero episodio di Sapia, spesso criticato per la sua "mediocrità" è, nella mia opinione, fra le pagine più sottili di Dante. Funziona come un buon esempio del "teatrale". Dopo lo spettacolo della cecità creato all'inizio del Canto ecco ga seduta e non percepisca nulla con la vista, come figura centrale del canto assume proporzioni drammatiche importanti. Le sue parole mostrano una certa rettorica declamativa. Sapia rivive drammaticamente il momento della sconfitta dei senesi. L'azione drammatica si lega paradossalmente all'immobilità fisica. Il lettore assimilando il testo visualizza gli avvenimenti storici. Mentre l'effetto di un dramma teatrale dipende in gran parte dalle capacità istrioniche dell'attore, Dante qui utilizza ogni possibilità linguistica e descrittiva per creare poeticamente un personaggio degno del palcoscenico di oggi.

La "performance" di Sapia, come già è stato detto, mostra come Dante sia creatore di una figura sospesa fra gesto e parola, fra mimo e recitazione. Come nel caso delle opere dei grandi scultori (penso al David di Bernini) il momento chiave è quello che precede l'azione, ed esso viene immortalato come preludio psicologico all'evento. Il mento levato, che indica la preparazione al discorso, crea anche l'impressione di una vecchia sdentata.

Il che contrasta con la figura storica di Sapia, che secondo i documenti ${ }^{\text {IO }}$ fu una nobildonna vissuta fra il I2 IO e il I278, moglie di Ghinibaldo Saracini

9. Figura fra storia e leggenda. Nato a Campi nel Chianti e morto nel I289, si crede all'età di centonove anni! Il pettine può avere una funzione simbolica. Si ricorda il detto popolare "ogni nodo viene al pettine", indicando il pettine come portatore della verità.

IO. Sanesi (1926); Tempesti (1936). Questa contiene un acconto di Sapia Ghibellina sposata con il Guelfo Ghinibaldo Saracini. Alla sconfitta del marito, concorrente per la posizione 
e zia paterna di Provenzal Salvani. Corre voce che per un certo periodo fu bandita da Siena. Se ciò fosse vero, una nuova luce illuminerebbe l'atteggiamento di Dante poeta fiorentino verso Sapia nobildonna senese, giacché il tema dell'esilio introdurrebbe esperienze in comune fra il poeta e la sua creazione drammatica/poetica. Ci sarebbe poi anche il tema della città medioevale $^{\text {II }}$. Il confronto Guelfo/Ghibellino che troveremo nel Canto successivo è toccato e introdotto qui in sordina. Mentre l'amore di Dante per la città natale si riflette nel ritratto di Farinata degli Uberti (If. X), che ha difeso Firenze dalla distruzione, in quello di Sapia è rappresentato il risentimento verso la propria città, che in questo caso è Siena. Ella infatti ricorda ai due pellegrini di avere pregato il Cielo affinché i suoi concittadini fossero sconfitti nella battaglia di Colle. Dunque sono qui due atteggiamenti contrastanti: uno di Amore per la propria città, impersonato dal personaggio di Dante, poeta fiorentino e pellegrino; l'altro di Odio, impersonato da Sapia nobildonna senese, peccatrice e anima penitente. Il legame tra i due personaggi è dato anche da un verso che contiene un riferimento all'età: "folle già discendendo l'arco d'i miei anni" in qualche modo riecheggia "Nel mezzo del cammin di nostra vita". Al tempo della battaglia di Colle Sapia aveva cinquantotto anni. Da ben ventitré anni aveva superato "l'arco degli anni", quando osò gridare contro Dio.

\section{DANTE REgista FRA DOMANDA E RISPOSTA}

La descrizione scenica, gli effetti sonori, l'immagine della memoria che si trasforma in recitazione e lo scambio di informazioni attraverso l'interrogazione e la conseguente confessione sono momenti chiave del Canto, il cui tono varia dalla descrizione geometrica della natura al ritratto dettagliato del personaggio di Sapia. Centrale all'episodio rimane la conversazione: sia quella di Virgilio le cui parole rimangono avvolte in un significato simbolico e didattico, che quella di Sapia nella quale si tocca il registro descrittivo, dimostrativo, penitenziale e ironico. In un breve spazio letterario Dante ha dato prova di una notevole varietà di toni e registri linguistici. Tenendo sempre presente la "teatralità" di questo canto, una lettura ad alta voce richiederebbe un'interpretazione capace di comunicare tutte le numerose sfumature del suo discorso. Nella parte finale del Canto la conversazione assume un tono più diretto con gli interrogativi di Sapia sulla persona di Dante e circa il viaggio che egli sta compiendo:

di Podestà di Siena, ella fu esiliata.Si veda anche Verbale della Repubblica di Siena del gennaio 1262.

II. Per uno studio sulla città e la cittadinanza medievali, e la sua presenza dell'opera dantesca si veda Honess (2006). 
Ma tu chi se', che nostro condizioni vai dimandando, e porti gli occhi sciolti, (Pg. XIII, I3O-I3I)

[...] "Chi t'ha dunque condotto Qua su tra noi, se giù ritornar credi?" (Pg. XIII, I39-I40)

Si noti il "dimandando" contenuto nella sua domanda, che conferisce un doppio senso di scambio drammatico alle parole cariche di curiosità. Dante non l'accontenta dicendole chi è, le svela invece il suo stato d'animo: cioè la sua paura per la colpa che si espia nella cornice successiva, quella degli orgogliosi, mentre nella cornice degli invidiosi spiega che il tempo da trascorrervi per lui sarà breve. Non nomina Virgilio. Il poeta latino viene indicato come "Costui... e non fa motto". Data l'età di Sapia, il nome di Dante non le avrebbe causato nessuna impressione. Ma oltre a questo, a me pare ci sia un altro motivo che può spiegare l'evasività di questa risposta. Dante e Sapia, come ho già indicato, rivendicano in modo conflittuale la loro appartenenza alle due città toscane. Ma ormai in Purgatorio questi argomenti non hanno più valore: "ciascuna è cittadina di una vera città". Così il fatto che Dante non riveli né il nome né la città d'origine è una conseguenza del suo rispetto per la legge del monte ${ }^{\mathrm{I} 2}$. Secondo una lettura più legata all'esperienza umana potrebbe sembrare un rifiuto a soddisfare una curiosità pettegola.

Dunque nel momento in cui si fa silenzio fra i personaggi di questo dramma politico e morale l'attenzione del lettore è attirata dal tema della cittadinanza di Dante. Il critico/lettore è portato a riflettere più in profondità sul tema della città e sul rifiuto di Dante di partecipare a una nuova battaglia di parole. La conversazione, l'interrogazione e lo scambio di informazioni diventano parte del dramma della parola in un canto pieno di ambiguità linguistica e di capovolgimenti letterari. L'orecchio della coscienza è sempre attento al non detto. L'incontro drammatico di due Toscani in terra neutrale, in un luogo e in un'atmosfera di penitenza spinge il critico a interpretare il canto come la drammatica espressione di un doppio confronto: fra il "visto" e il "non visto" e fra il "detto" e il "non detto". Siamo di fronte agli elementi fondamentali della "teatralità" dantesca: immagine e parola, oscurità e silenzio. All'inizio del canto il silenzio del luogo predomina. Alla fine, si manifesta il rifiuto della parola. Così il primo poeta italiano dopo più di sette secoli apre a un nuovo vasto pubblico un teatro di azione storica fatta di confronti verbali, di contrasti astratti e di alternative politiche e intellettuali sul palcoscenico della giustizia divina.

I2. Si ricorda un atteggiamento del tutto diverso riguardante la rivelazione d'identità di Bocca degli Abati in If. XXXII. 


\section{BiBLIOGRAFIA}

Audeh, A., and Havely, N., 20I2, Dante in the Long Nineteenth Century, New York, Oxford University Press.

Bentley, E.,1965, The Life of the Drama, London, Methuen.

Alighieri, D., La divina commedia Purgatorio, a cura di Bosco, U., E Reggio, G., 2002, Firenze, Le Monnier.

Della Terza, D., 20I3, "Il primo Canto dell'Invidia: Purgatorio XIII", in Id., Dante e noi-Scritti danteschi, Roma, Edicampus, pp. I4I-I57 [già in Filologia e critica, I7, I992, pp. 3-2I].

Donadoni, E.,I963, "Le tre donne della Commedia", in Studi danteschi e manzoniani, Binni, W. (a cura di), Firenze, La nuova Italia, pp. 75-99.

Gardner, E., I9I3, Dante and the Mystics, London, Dent.

Getto, G., 1964, Letture dantesche, 2, Firenze, Sansoni, pp. 260-26I.

Gilson, S., 2000, Medieval Optics and Theories of Light in the Works of Dante, Lamperte, Edwin Mellen Press.

Glenn, D., 2005, "The Envious Eye: Echoes of Inferno XIII in Purgatorio XIII, the Figures of Pier della Vigna and Sapia", in Flinders Dante Conferences (2002-2004), Lythrum Press, Flinders University, Adelaide, pp. 65-76.

-, 2008, Dante's Reforming Mission and Women in the Comedy, Leicester, Trobador.

Heer, F., I96I, The Medieval World, New York, Barnes and Noble.

Honess, C., 2006, From Florence to the Heavenly City, Oxford, Legenda.

Jolivet, R., I933, Dieu soleil des esprits, Paris, Desclée de Brouwer.

Mazzeo, J. A., I960, Medieval Cultural Tradition in Dante's Comedy, New York, Cornell University Press.

Mazzotta, G., 1993, Dante's Vision of the Circle of Knowledge, Princeton, Princeton University Press.

Moevs, C., 2005, The Metaphysics of Dante's Comedy, Oxford/New York, Oxford University Press.

McEvoy, J., 1982, The Philosophy of Robert Grosseteste, Oxford.

Mounin, G.,1985, Semiotic Praxis Studies in Pertinence and in the Means of Expression and Communication, New York/London.

O'Grady, D., I987, "Women Damned, Penitent and Beatified in the Divine Comedy", in Dante Readings, Haywood, E., (ed.), Dublin, Foundation for Italian Studies University College Dublin, pp. 73-Io6.

—, 2003, "Francesca da Rimini from Romanticism to Decadence", in E. Haywood (ed), Dante Metamorphoses Episodes in a Literary Afterlife, Dublin, Four Courts Press, pp. 22I-239;

—, 2013, "A Dantesque Dualism: Francesca da Rimini between Temptation, Betrayal, and Perturbation", in M. Ciavolella; F. Farina (ed.) Women in Hell, Rimini, Romagna Arte e Storia, pp. 73-82.

Pernicone, V., (a cura di), 1946, [F.Sacchetti] Trecento novelle, Firenze, Sansoni.

Petrocchi, G., 1983, Vita di Dante, Bari, Laterza.

Rossi-Landi, F., I979, "Il teatro come azione sociale", in Id., Semiotica e ideologia, Milan, Bompiani, pp. 43-45.

Sanesi, I., I923, "Sapia", Studi danteschi, 6, p. IoI.

Santini, E., I964, "Il canto XIII del Purgatorio", in Getto, G. (ed.), Letture dantesche, vol. 2 Purgatorio, Firenze, Sansoni, pp. 260-26r. 
Shapiro, M., 1975, Women Earthly and Divine in the Comedy of Dante, Kentucky, Lexington.

Singleton, G. S., I970, "La cecità degli invidiosi", in G. Barberi Squarotti; A. Jacomuzzi (ed.), Critica dantesca, Torino, pp. 366-369.

Tempesti, F., 1936, "Provenzal Salvani", Bulletino senese di storia patria, n. s., vii.

Yunck, J. A., 1963, The Lineage of Lady Meade. The Development of Medieval Venality Satire, Notre Dame. 
